\title{
Qualität in der ambulanten Pflege
}

\author{
Andreas Büscher und Moritz Krebs \\ (c) Der/die Autor(en) 2018 \\ K. Jacobs et al. (Hrsg.), Pflege-Report 2018 \\ https://doi.org/10.1007/978-3-662-56822-4_11
}

\section{Zusammenfassung}

Die Entwicklung und Sicherstellung pflegerischer Qualität wird nicht erst seit der Einführung der Pflegeversicherung intensiv diskutiert. Im Zuge dieser Auseinandersetzung zeigt sich, dass insbesondere in der ambulanten Versorgung unterschiedliche Ansichten darüber bestehen, wie Pflegequalität zu definieren, zu entwickeln und zu sichern ist. Dieser Beitrag stellt daher zentrale Merkmale sowie Rahmenbedingungen ambulanter Pflege dar, zeigt auf, welche unterschiedlichen Formen der Pflege und Unterstützung in der häuslichen Versorgung zum Tragen kommen und wie die Diskussion um die Qualität der ambulanten Pflege durch die unterschiedlichen Perspektiven der beteiligten Akteure bestimmt wird. Daran anschließend werden Ansatzpunkte für eine weitere Diskussion um die Qualität in der ambulanten Pflege aufgezeigt.

The development and assurance of the quality of nursing care has been discussed intensively before the introduction of long-term care insurance. The course of this debate revealed differing opinions of how to define, develop and assure nursing care quality, particularly in home- and community-based care. This chapter summarizes core aspects and legislative frameworks of home and community care in Germany. It outlines the varying forms of nursing care and other types of support in home care and describes how the discussion on nursing care quality is influenced by different stakeholders' perspectives. Finally, approaches to developing and assuring the quality of professional home care providers will be discussed.

\subsection{Einleitung}

Der Vorrang der ambulanten vor einer stationären Versorgung gilt seit langem als gesundheits- und sozialpolitische Maxime und trifft den Wunsch der meisten Menschen, im Fall von Krankheit und Pflegebedürftigkeit so lange wie möglich in der häuslichen Umgebung zu verbleiben. Vor diesem Hintergrund wäre zu erwarten, dass es eine intensive Auseinandersetzung um die Qualität der häuslichen Versorgung gibt und die Vorstellungen darüber, wie eine wünschenswerte häusliche Versorgungskonstellation beschaffen sein sollte und welche Rolle professionelle ambulante Pflegedienste darin spielen können, deutlich ausgeprägt sind. Auch wenn sich hierzu im Zuge der Weiterentwicklung der Qualitätsprüfungen im SGB XI (vgl. $\$ \$ 114 \mathrm{ff}$ SGB XI) durchaus Anzeichen wiederfinden, bezieht sich die Diskussion zur Qualität der Pflege dennoch im Wesentlichen auf die institutionalisierte Versorgung in Krankenhäusern und Pflegeheimen.

Einer der Gründe für die nachrangige Betrachtung der Qualität der ambulanten Pflege ist sicherlich, dass die Zuschreibung einer professionellen Verantwortung für die Versorgungsqualität leichter fällt, wenn die professionellen Akteure eine tatsächliche Steuerungsverantwortung haben und das Geschehen maßgeblich beeinflussen können. Dies ist in Krankenhäusern und Pflegeheimen, in denen sich die Adressaten der Pflege rund um die Uhr auf- 
halten, der Fall. Hinzu kommt, dass das institutionelle Umfeld in der Regel im Sinne professioneller Versorgungsprozesse gestaltet ist. In der ambulanten Pflege bestehen jedoch umgekehrte Voraussetzungen. Die professionellen Akteure sind nur in zeitlich äußerst begrenzter Form in häuslichen Pflegearrangements präsent. Dort ist das Umfeld in erster Linie nach individuellen Möglichkeiten und Vorlieben der Haushaltsmitglieder und nicht unter pflegepraktischen Gesichtspunkten gestaltet. Diese und noch weitere Charakteristika der ambulanten Pflege haben eine Auswirkung auf die Qualität der Versorgung. Sie werden im folgenden Beitrag skizziert, bevor Ansatzpunkte für die zukünftige Diskussion um die Qualität in der ambulanten Pflege aufgezeigt werden.

\subsection{Charakteristika der ambulanten Pflege}

Ein wesentliches Ziel der ambulanten Pflege besteht darin, Menschen, die wegen einer Krankheit, Beeinträchtigung oder Behinderung der Unterstützung durch andere Personen bedürfen, den Verbleib in der eigenen Wohnung zu ermöglichen. Ambulante Pflegedienste verfolgen dieses Ziel jedoch nicht allein, sondern sind Teil eines Gesamtumfeldes der häuslichen Pflege, in dem vor allem die familiale Pflege den größten Beitrag leistet, aber auch weitere Akteure involviert sind. Die Qualität der häuslichen Pflege kann daher nicht auf die Qualität der ambulanten Pflegedienste reduziert werden und liegt auch nicht allein in ihrer Verantwortung. Vielmehr bedeutet häusliche Pflege, innerhalb eines ausbalancierten und leistbaren Kontinuums gesundheitliche und soziale Bedürfnisse von Menschen in ihrer häuslichen Umgebung durch angemessene und qualitativ hochwertige gesundheitliche und soziale Dienste durch formell und informell Pflegende unter Anwendung von Technologie zu befriedigen (WHO 2008).

Diese Sichtweise macht zwei wichtige Aspekte der ambulanten pflegerischen Versorgung deutlich: Sie ist weder eindeutig dem Gesundheitswesen noch dem Sozialwesen zuzuordnen, sondern sieht sich der Herausforderung ausgesetzt, sowohl zur gesundheitlichen wie auch zur sozialen Versorgung beizutragen. Dieser Zwiespalt zieht sich auch durch ihre historische Entwicklung, in der mal die lebensweltliche Alltagsbegleitung, mal die Unterstützung und Sicherstellung von Diagnostik und Therapie die wesentlichen politischen Zielsetzungen bildeten (Moers 1997; Hackmann 2001). Zum anderen erfolgt häusliche Pflege sowohl auf formeller wie auf informeller Basis. Die informelle Pflege wird im Wesentlichen von pflegenden Familienangehörigen erbracht. Dem informellen Sektor steht der formelle Pflegesektor gegenüber, in dem die Pflege im Rahmen bezahlter Arbeitsverhältnisse erbracht wird und zu dem vor allem die ambulanten Pflegedienste zählen. Daneben gibt es eine zunehmende Anzahl anderer Anbieter, z. B. von pflegebegleitenden oder haushaltsnahen Dienstleistungen, die ebenfalls dem formellen Sektor zuzurechnen und in häusliche Pflegearrangements eingebunden sind. Nicht zu ignorieren sind darüber hinaus die Hilfen, die eher einer Grauzone zuzurechnen sind, aber in der Diskussion um die ambulante pflegerische Versorgung nicht ignoriert werden können: die Hilfen, die von Frauen aus vorwiegend osteuropäischen Ländern in deutschen Pflegehaushalten erbracht werden (Larsen et al. 2009; Neuhaus et al. 2009).

Aus der problematischen Zuordnung zwischen Gesundheits- und Sozialwesen sowie dem Verhältnis zwischen formeller und informeller Pflege erwachsen einige Herausforderungen und Spannungsfelder, die in der bisherigen Diskussion um die Qualität der ambulanten Pflege bislang nur unzureichend Berücksichtigung gefunden haben, z. B.

- hinsichtlich der Frage der familialen gegenüber der öffentlichen Verantwortung (ausgeübt durch die Pflegedienste),

- die Abgrenzung und Zuständigkeit zwischen formeller und informeller Pflege,

- die Entscheidung, ob es eine lokale oder nationale Verantwortung gibt und ob diese durch die Regierung, den Markt oder Verbände im Rahmen von Selbstverwaltung wahrgenommen wird und

- hinsichtlich der Wahl- und Wunschrechte der Menschen im Sinne der individuellen Selbstbestimmung gegenüber einem professionellen Diktat (Burau et al. 2007). 


\subsection{Häusliche Pflegearrangements}

Bevor die Qualität professioneller ambulanter Pflegedienste eingehender betrachtet wird, soll im Folgenden kurz der Blick auf weitere Formen der Pflege und Unterstützung geworfen werden. Die Situation in den Pflegehaushalten, der Zeitpunkt und der Grund der Inanspruchnahme professioneller Hilfe, die Wünsche der pflegebedürftigen Menschen und ihrer Angehörigen und ihr Kenntnisstand zur Pflegesituation sind in hohem Maße heterogen und durch lebensweltliche Aspekte charakterisiert. Die Diskussion über die Bedeutung der Lebenswelt pflegebedürftiger Menschen wurde bereits in den 1990er Jahren intensiv geführt (Braun und Schmidt 1997), geriet jedoch wieder in Vergessenheit. Bei chronischer Krankheit und Pflegebedürftigkeit verändert sich die Lebenswelt der beteiligten Personen erheblich, insbesondere in Bezug auf die Möglichkeiten, sich im Raum zu bewegen, Zeit und soziale Beziehungen zu gestalten sowie hinsichtlich der Vorstellungen über Alltagsorganisation und -bewältigung (Schaeffer und Moers 2011).

In einem lebensweltlichen Verständnis steht daher auch weniger die Frage der Qualität der Pflege im Vordergrund, sondern die eines gelingenden Alltags. Verantwortlich für dieses Gelingen sind nicht primär einzelne Akteure, sondern das gesamte Setting und die Lebenswelt, die den Alltag darstellt. Dazu gehören die informellen, familialen Helfer ebenso wie die formellen Dienstleister. Den Anteil des informellen Hilfesystems an der Herstellung des Alltags, an der Bewältigung von Krankheit, Behinderung und Pflegebedürftigkeit sichtbar zu machen und die familialen Helfer dazu zu qualifizieren, sollte eine der wesentlichen Aufgaben sein, mit denen sich professionelle Helfer aber seit jeher schwer tun, da sie sich in Konkurrenz sehen oder den Beitrag der informellen Pflege für selbstverständlich halten (Zeman 1997; Büscher 2007). Wünschenswert wäre demgegenüber eine familienorientierte Unterstützung, durch die das gesamte Pflegearrangement stabilisiert und bestärkt wird.

Durch die Einschaltung eines Pflegedienstes wird aus einem vormals informellen ein gemischtes Pflegearrangement, in dem formelle und informelle Helfer eingebunden sind. Ein Blick in die Pflegesta- tistik (Statistisches Bundesamt 2017) verdeutlicht, dass sich die Zahl der Haushalte vergrößert, in denen neben pflegenden Angehörigen auch ambulante Pflegedienste Leistungen erbringen. Das Verhältnis von formeller und informeller Pflege ist dabei keineswegs spannungsfrei und die Einschaltung formeller Pflege bringt nicht immer eine Stabilisierung des Gesamtarrangements oder eine Verbesserung der Pflegesituation mit sich (WardGriffin und McKeever 2000).

Nicht vergessen werden darf in der Betrachtung die Perspektive des pflegebedürftigen Menschen, der den Ausgangspunkt der Begegnung zwischen formeller und informeller Pflege bildet. Ein Ansatz zum Verständnis der komplexen Beziehungen in häuslichen Pflegearrangements ist der "six senses framework" nach Nolan et al. (2001), in dem der Versuch unternommen wird, wesentliche Kriterien zur Gestaltung eines häuslichen Pflegearrangements aus der Perspektive des pflegebedürftigen Menschen, der pflegenden Familienangehörigen und der Mitarbeiter formeller Dienste zu beschreiben. Die »six senses« umfassen:

- Sicherheit hinsichtlich der essenziellen physiologischen und psychologischen Bedürfnisse sowie vor Bedrohung, Schaden oder Schmerz,

- Kontinuität in der persönlichen Biografie und eine konsistente Versorgung innerhalb etablierter Pflegebeziehungen durch bekannte Pflegepersonen,

- Zugehörigkeit durch die Bildung und/oder Aufrechterhaltung bedeutsamer und gegenseitiger Beziehungen,

- Zielgerichtetheit hinsichtlich der Fähigkeit, Ziele und Herausforderungen zu identifizieren sowie Ermessensspielraum für eigene Entscheidungen zu haben,

- Etwas erreichen können im Hinblick auf bedeutsame Ziele sowie

- Bedeutsamkeit im Sinne der Anerkennung und Wertschätzung als Person und des Gefühls der Wichtigkeit der eigenen Existenz und Handlungen. 


\subsection{Rahmenbedingungen ambulanter Pflegedienste}

Der Beitrag ambulanter Pflegedienste in häuslichen Pflegearrangements erfolgt vor dem Hintergrund festgelegter gesetzlicher Rahmenbedingungen. Um ihre Leistungen mit den Pflegekassen abrechnen zu können, benötigen sie einen Versorgungsvertrag, dessen Abschluss u. a. an die Bedingung gekoppelt ist, dass die Dienste eine Gewähr für eine leistungsfähige und wirtschaftliche pflegerische Versorgung bieten und sich verpflichten, ein einrichtungsinternes Qualitätsmanagement umzusetzen (vgl. $\$ 112$ SGB XI). Darüber hinaus sind im SGB XI Regelungen hinsichtlich der Qualitätssicherung und Qualitätsprüfung festgeschrieben ( $\$ \$ 113 \mathrm{ff}$ SGB XI).

Eine wichtige Rolle für die Tätigkeit ambulanter Pflegedienste spielen die Möglichkeiten, die eigenen Leistungen abzurechnen. Diese orientieren sich nach $\$ 36$ SGB XI am Begriff der Pflegebedürftigkeit. Nach der Einführung des neuen Begriffs der Pflegebedürftigkeit zum 01.01.2017 steht jedoch eine Anpassung der entsprechenden Rahmenvereinbarungen an diesen erweiterten Begriff zum Ende des Jahres 2017 noch aus. So besteht weiterhin ein verrichtungsorientiertes Verständnis professioneller Pflege, was zum einen zu einer systembedingten Einengung des professionellen Handlungsspielraums führt, zum anderen den Pflegebedürftigen und ihren Angehörigen vermittelt, dass es sich bei der "Dienstleistung Pflege« um ein Produkt handelt, das individuell, einem Baukastenprinzip ähnlich, zusammengestellt werden kann (Büscher et al. 2005). Die Wirklichkeit vieler häuslicher Pflegearrangements gestaltet sich jedoch deutlich komplexer und bedarf einer über Alltagsverrichtungen hinausgehenden Unterstützung.

Die meisten ambulanten Pflegedienste erbringen darüber hinaus auch Leistungen der häuslichen Krankenpflege nach $₫ 37$ SGB V, die ärztlich verordnet werden müssen. Dies geschieht auf der Grundlage von Richtlinien, die vom Gemeinsamen Bundesausschuss der Ärzte und Krankenkassen nach $\$ 92$ SGB V beschlossen werden (G-BA 2017).

In der Versorgungspraxis zeigt sich, v. a. auf Leitungsebene, sehr deutlich, dass die Frage, wie etwas finanziert werden kann, Vorrang gegenüber der Überlegung hat, was in einer gegebenen Situation gut oder angemessen wäre (Slotala 2011). Überlegungen zur Qualitätsentwicklung befinden sich somit in einem dauerhaften Zielkonflikt mit Wirtschaftlichkeitserwägungen. Die ambulanten Pflegedienste agieren unter einem erheblichen Problemdruck, der sich auf Aspekte der Wirtschaftlichkeit und Marktentwicklung, die Personalsituation sowie die Strukturen und das Spektrum der Leistungserbringung bezieht (Büscher und Horn 2010).

Vor diesem Hintergrund bezieht sich einer der Diskussionspunkte im bestehenden System der Qualitätsprüfungen und Qualitätsberichterstattung darauf, diese in Übereinstimmung mit dem vorhandenen Leistungsspektrum zu gestalten und nicht durch die Qualitätsprüfungen von einem Qualitätsniveau auszugehen, das im geltenden Leistungsrecht keine Entsprechung findet.

\subsection{Qualität der ambulanten Pflege}

Was bedeuten die Rahmenbedingungen und Charakteristiken nun für die Diskussion um die Qualität der ambulanten Pflege? Im Rahmen einer Perspektivenwerkstatt des Zentrums für Qualität in der Pflege (ZQP) wurde eine neue Ordnung für die Qualitätsdiskussion anhand getrennt zu betrachtender und zu diskutierender Bereiche vorgeschlagen (ZQP 2013; Büscher und Klie 2013):

- Qualität professionellen Pflegehandelns dahinter verbirgt sich eine Schärfung des fachlichen Profils der ambulanten Pflege hinsichtlich zukünftiger Aufgaben, die sich aus dem erweiterten Verständnis des neuen Begriffs der Pflegebedürftigkeit ergeben und die Grundlage für eine Erweiterung des derzeit begrenzten Leistungsspektrums sind.

- Partizipative Aushandlung und Vereinbarung von Zielen und Maßnahmen in Hilfeplänen Ausmaß und Inhalt ambulanter Pflege kommunikativ auszuhandeln und sich darüber zu verständigen ist angesichts oftmals unterschiedlicher Vorstellungen der Angehörigen eines Pflegehaushalts und der Mitarbeiter eines professionellen Pflegedienstes notwendig. Die letztliche Entscheidung liegt beim pflegebedürftigen Menschen, jedoch ist es Aufgabe der professionellen Dienste, die aus fachlicher 
Sicht wichtigen Aspekte der Situation aufzuzeigen, ein entsprechendes Unterstützungsangebot zu unterbreiten und somit die Entscheidung zu unterstützen.

- Individuelle Pflegebedürftigkeit und Teilhabe als Ausgangspunkt zur Einschätzung von Pflegequalität - dieser Aspekt verweist darauf, in Zukunft die Qualität der Pflege nicht nur institutionell (also mit Bezug auf professionelle Leistungserbringer) zu betrachten, sondern auch die individuelle Situation der pflegebedürftigen Menschen in den Blick zu nehmen, Veränderungen zu erfassen und anschließend zu prüfen, ob Veränderungen die Konsequenz fortschreitender Krankheitsprozesse, individueller Entscheidungen oder unzureichender professioneller Versorgung sind.

- Objektive und subjektive Parameter der Lebensqualität - Aspekte des Wohlbefindens und der Sicherheit haben eine entscheidende Bedeutung für die subjektive Wahrnehmung der Qualität der Versorgung. Ihre Sicherstellung ist eine schwierige Aufgabe, die über externe Qualitätsprüfungen nur bedingt sichergestellt werden kann.

- Lokale Infrastruktur - die Lebensqualität pflegebedürftiger Menschen kann nicht losgelöst von ihrer lokalen Umwelt betrachtet werden und ist in hohem Maße von dieser bestimmt. Entsprechend sollten Fragen des Lebens mit Pflegebedürftigkeit und der Qualität häuslicher Versorgung Themen auf der lokalen und kommunalpolitischen Agenda sein.

Vor dem Hintergrund dieser Überlegungen bezieht sich die Diskussion um die Qualität der ambulanten Pflege vor allem auf die Qualität professionellen Pflegehandelns und die partizipative Aushandlung und Vereinbarung von Zielen und Maßnahmen. Das professionelle Pflegehandeln und der Verantwortungsbereich ambulanter Pflegedienste werden vorrangig durch die vertraglichen Vereinbarungen zwischen Pflegedienst und Pflegehaushalt bestimmt. Es kann jedoch aus fachlichen Erwägungen erwartet werden, dass insbesondere zu Beginn einer Pflegesituation eine umfassende Einschätzung der Situation und eine entsprechende Kommunikation mit Pflegebedürftigen und Angehörigen stattfindet. Sollte es im Verlauf der Pflegesituation Veränderungen geben, die eine fachliche Bewertung erfordern (wie z. B. Verschlechterungen der Gesamtsituation, Neuauftreten von Risiken), sollte seitens des Pflegedienstes darauf eingegangen werden. Leistungsrechtlich sollten Möglichkeiten geschaffen werden, begründete, über die vereinbarten Leistungen hinausgehende Maßnahmen mit den Kostenträgern abrechnen zu können.

Die Frage der Verantwortung bezieht sich darüber hinaus auch auf die unterschiedlichen Regelungen in SGB V und SGB XI. Während die Verantwortung für die Einschätzung der Situation im SGB XI weitgehend dem ambulanten Pflegedienst obliegt, erfolgt dies für Leistungen im SGB V im Vorfeld durch den verordnenden Arzt. Diese Situation ist so lange unter Qualitätsgesichtspunkten unproblematisch, wie es zwischen Pflegedienst, Pflegehaushalt und behandelndem Arzt eine weitgehende Übereinstimmung in der Einschätzung der anzugehenden Problemlagen gibt. Ist dies nicht der Fall, sind Konflikte programmiert. Ein möglicher Weg wäre die Übertragung ärztlicher Tätigkeiten auf Berufsangehörige der Alten- und Krankenpflege (G-BA 2011). Ob diese Auseinandersetzung zukünftig berufsrechtlich oder leistungsrechtlich geführt werden wird, lässt sich nur bedingt prognostizieren. Für ambulante Pflegedienste können daraus jedoch in beiden Fällen Handlungsoptionen oder Handlungsdruck entstehen.

Zeichnen im SGB XI die Vertragsparteien nach $\$ 113$ SGB XI für die Maßstäbe und Grundsätze verantwortlich, so ist es innerhalb des SGB V der Gemeinsame Bundesausschuss, der die maßgeblichen Bestimmungen zur Qualitätssicherung erlässt. Angesichts der Größenordnung, in der ambulante Pflegedienste Leistungen nach SGB V erbringen, erscheint es zumindest bemerkenswert, dass die maßgeblichen Qualitätsbestimmungen vorrangig innerhalb der Gremien des SGB XI diskutiert und vereinbart werden. Hier besteht eine strukturelle Schieflage, über die es sich vertiefend nachzudenken lohnt.

Da die qualitätsrelevanten Pflegeprobleme der Nutzer ambulanter Pflegedienste oftmals sektorübergreifenden Charakter haben, ist es angezeigt, bei Fragen der Qualitätsentwicklung die unter- 
schiedlichen Ansätze aus dem Gesundheitswesen und der pflegerischen Versorgung zu berücksichtigen. Für die weiteren Auseinandersetzungen bedeutet das, nicht nur im Rahmen der ärztlichen Versorgung, sondern auch in der Pflege sektorübergreifende Verfahren der Qualitätssicherung zu entwickeln. Da es hierzu derzeit jedoch kaum Ansatzpunkte gibt, auf denen sich aufbauen ließe, sollte im Zuge der Weiterentwicklung der Qualitätssicherung in der ambulanten Pflege die Diskussion mit hausärztlichen Vertretern und den Akteuren der Selbstverwaltung im SGB V geführt werden.

Die Qualität in der Pflege ist das Ergebnis von Prozessen zwischen Leistungserbringer und Adressat der pflegerischen Handlungen. Für die Adressaten der Leistungen eines Pflegedienstes ist es zudem vorrangig, wie sie die Qualität in ihrer jeweiligen Situation wahrnehmen. Daher ist neben den strukturellen Aspekten der Qualität des professionellen Pflegehandelns auch die fachliche Weiterentwicklung entscheidend. Dies macht es erforderlich, Ziele in Form von Standards, Leitlinien, Handlungsempfehlungen o. ä. festzulegen. Entsprechend sollte es auch für ambulante Pflegedienste selbstverständlich sein, sich mit den Ergebnissen des eigenen Handelns auseinanderzusetzen, diese zu bewerten und auf Basis der Ergebnisse das eigene Handeln ggf. neu auszurichten. Dabei kommt dem internen Qualitätsmanagement eine vorrangige Bedeutung zu. Es ist eine der Grundannahmen der Qualitätslehre, dass Qualität nicht von außen in eine Organisation hineingeprüft werden kann, sondern dass diese intern hergestellt werden muss. Entsprechend sollten Leistungserbringer darum bemüht sein, ihre Prozesse so zu gestalten, dass formulierte Qualitätsziele erreicht werden können.

Ein Blick in die nationale und internationale Literatur bringt eine Vielzahl möglicher Themen und Ansatzpunkte für die Qualitätsentwicklung in der ambulanten Pflege hervor. Neben den bereits erwähnten »six senses" werden Fragen der Sicherheit auch von anderen Autoren adressiert und dienen als Überbegriff für unterschiedliche Themen wie z. B. den Umgang mit Medikamenten. Angesprochen werden darüber hinaus Fragen eines umfassenden und angemessenen Assessments der Situation, der Versorgungskoordination, Patientenedukation im Sinne einer Förderung des Selbst- managements, der Kompetenz der professionellen Dienstleister und der individuellen Lebensqualität (Leff et al. 2015). Auch Aspekte des Zugangs und der generellen Verfügbarkeit ambulanter Pflegeleistungen sowie ihrer Effektivität, Koordination, Angemessenheit, Kontinuität und Patientenzentriertheit, Rechtzeitigkeit und Nachhaltigkeit werden diskutiert. Nicht zuletzt konnten Hinweise auf die Bedeutung der Stabilisierung häuslicher Pflegearrangements und der Beratung und Edukation für die Qualität der ambulanten Pflege identifiziert werden (Hasseler et al. 2013).

Es fehlt also nicht an möglichen Bereichen, Dimensionen und Kriterien, anhand derer Qualitätsfragen der häuslichen Pflege insgesamt und der Qualität der ambulanten Pflegedienste im Besonderen konkretisiert werden könnten. Eine Rangfolge der verschiedenen Ergebnisbereiche dürfte jedoch nur schwerlich zu begründen sein, da ihre Wichtigkeit in hohem Maße von der individuellen Situation des pflegebedürftigen Menschen abhängt. Es spricht jedoch nichts dagegen, innerhalb eines ambulanten Pflegedienstes oder eines Verbandes einzelne Bereiche herauszugreifen und ihnen verstärkte Aufmerksamkeit zukommen zu lassen. Hier bieten sich natürlich auch die Bereiche an, zu denen bereits Qualitätsinstrumente vorliegen. Somit lässt sich die Aufmerksamkeit auf zentrale Bereiche lenken, die Kompetenz der Mitarbeiterinnen und Mitarbeiter erhöhen und die leistungsrechtliche Verankerung voranzutreiben.

\subsection{Fazit}

Es konnte gezeigt werden, dass die Diskussion um die Qualität der ambulanten Pflege eine Vielzahl von Facetten umfasst. Im Rahmen der Entwicklung der zukünftigen Verfahren für Qualitätsprüfungen nach $₫ 114$ SGB XI und die Qualitätsdarstellung nach $\$ 115$ Abs. 1a SGB XI in der ambulanten Pflege wird die Qualität des professionellen Pflegehandelns im Mittelpunkt stehen. Dabei ist es erstrebenswert, dass die Maßgaben des externen Prüfgeschehens die richtigen Impulse für die interne Qualitätsentwicklung in den ambulanten Pflegediensten geben und es somit zu einer stärkeren Harmonisierung zwischen Prüfung und dem alltäglichen Pflege- 
geschehen kommt. Die durch die Pflege-Transparenzvereinbarung (PTV-A) bedingte dokumentationsorientierte Prüfung hat den Blick eher auf die Dokumentation als auf die Gestaltung von Pflegeprozessen gerichtet.

Darüber hinaus sollte jedoch auch der nicht durch ambulante Pflegedienste geleisteten Pflege im häuslichen Umfeld verstärkte Aufmerksamkeit gewidmet werden. Die Pflege durch Familienan- gehörige stellt nach wie vor den größten Teil der pflegerischen Versorgung in Deutschland sicher. Sie zu unterstützen, zu flankieren und eine Prävention gegenüber Fehlentwicklungen zu leisten, die zur Überlastung pflegender Angehöriger oder zu problematischen Konstellationen führen können, ist eine wichtige Aufgabe, um die Qualität der häuslichen Versorgung mittel- und langfristig zu sichern.

Open Access Dieses Kapitel wird unter der Creative Commons Namensnennung 4.0 International Lizenz (http://creativecommons. org/licenses/by/4.0/deed.de) veröffentlicht, welche die Nutzung, Vervielfältigung, Bearbeitung, Verbreitung und Wiedergabe in jeglichem Medium und Format erlaubt, sofern Sie den/die ursprünglichen Autor(en) und die Quelle ordnungsgemäß nennen, einen Link zur Creative Commons Lizenz beifügen und angeben, ob Änderungen vorgenommen wurden.

Die in diesem Kapitel enthaltenen Bilder und sonstiges Drittmaterial unterliegen ebenfalls der genannten Creative Commons Lizenz, sofern sich aus der Abbildungslegende nichts anderes ergibt. Sofern das betreffende Material nicht unter der genannten Creative Commons Lizenz steht und die betreffende Handlung nicht nach gesetzlichen Vorschriften erlaubt ist, ist für die oben aufgeführten Weiterverwendungen des Materials die Einwilligung des jeweiligen Rechteinhabers einzuholen.

\section{Literatur}

Braun U, Schmidt R (Hrsg) (1997) Entwicklung einer lebensweltlichen Pflegekultur. Transfer-Verlag, Regensburg

Burau V, Theobald H, Blank RH (2007) Governing Home Care. A Cross-National Comparison. Edward Elgar, Cheltenham (UK)

Büscher A, Boes C, Budroni H, Hartenstein A, Holle B (2005) Finanzierungsfragen der häuslichen Pflege. Eine qualitative Untersuchung zur Einführung personenbezogener Budgets. Abschlussbericht. Private Universität Witten/ Herdecke, Witten

Büscher A (2007) Negotiating helpful action. A susbtantive theory of the relationship between formal and informal care. Acta Universitatis Tamperensis 1206. Tampere University Press, Tampere

Büscher A, Horn A (2010) Bestandsaufnahme zur Situation in der ambulanten Pflege. Ergebnisse einer Expertenbefragung. P10-145. Veröffentlichungsreihe des Instituts für Pflegewissenschaft an der Universität Bielefeld. IPW, Bielefeld

Büscher A, Klie T (2013) Perspektivenwerkstatt 2013. Qualitätsentwicklung und Lebensweltorientierung in der häuslichen Pflege. Abschlussbericht für das ZQP. ZQP, Berlin

G-BA (Gemeinsamer Bundesausschuss) (2011) Beschluss des Gemeinsamen Bundesausschusses über eine Richtlinie über die Festlegung ärztlicher Tätigkeiten zur Übertragung auf Berufsangehörige der Alten- und Krankenpflege zur selbständigen Ausübung von Heilkunde im Rahmen von Modellvorhaben nach § 63 Abs. 3c SGB V
G-BA (Gemeinsamer Bundesausschuss) (2017) Richtlinie des Gemeinsamen Bundesausschuss über die Verordnung von häuslicher Krankenpflege (Häusliche Krankenpflege Richtlinie) in der Neufassung vom 17. September 2009, zuletzt geändert am 16. März 2017. Veröffentlicht im Bundesanzeiger BAnz AT 01.06.2017 B3

Hackmann M (2001) Zur Geschichte der Gesundheitsförderung in der ambulanten Pflege. In: Gehring M, Kean S, Hackmann M, Büscher A. Familienbezogene Pflege. Huber, Bern, S 209-219

Hasseler M, Görres S, Fünfstück M (2013) Indikatoren zur Messung von Struktur-, Prozess- und Ergebnisqualität sowie Lebensqualität in der ambulanten pflegerischen Versorgung. Expertise im Auftrag des GKV-Spitzenverbandes. GKV-Spitzenverband, Berlin

Larsen C, Joost A, Heid S. (Hrsg) (2009) Illegale Beschäftigung in Europa. Die Situation in Privathaushalten älterer Personen. Rainer Hampp Verlag, München

Leff B, Carlson CM, Saliba D, Ritchie C (2015) The invisible Homebound: Setting quality-of-care standards for home-based primary and palliative care. Health Affairs; 34(1):21-29

MDS (Medizinischer Dienst des Spitzenverbandes Bund der Krankenkassen) (2014) 4. Pflege-Qualitätsbericht des MDS nach $\S 114 a$, Abs. 6 SGB XI. Qualität in der ambulanten und stationären Pflege. MDS, Essen

Moers M (1997) Ambulante Pflege in Deutschland - auf dem Weg zur Gemeinwesenorientierung? Pflege; 10(2):101112

Neuhaus A, Isfort M, Weidner F (2009) Situation und Bedarfe von Familien mit mittel- und osteuropäischen Haus- 
haltshilfen. Deutsches Institut für angewandte Pflegeforschung, Köln

Nolan M, Davies S, Grant G (Hrsg) (2001) Working with older people and their families. Key issues in policy and practice. Open University Press, Buckingham

Schaeffer D, Ewers M (Hrsg) (2002) Ambulant vor stationär. Perspektiven für eine integrierte ambulante Pflege Schwerkranker. Huber, Bern

Schaeffer D, Moers M (2011) Bewältigung chronischer Krankheiten - Herausforderungen für die Pflege. In: Schaeffer D, Wingenfeld K (Hrsg) Handbuch Pflegewissenschaft. Juventa, Weinheim, S 329-364

Slotala L (2011) Ökonomisierung der ambulanten Pflege. Eine Analyse der wirtschaftlichen Bedingungen und deren Folgen für die Versorgungspraxis ambulanter Pflegedienste. Springer VS, Wiesbaden

Statistisches Bundesamt (2017) Pflegestatistik 2015. Pflege im Rahmen der Pflegeversicherung. Deutschlandergebnisse. Wiesbaden

Ward-Griffin C, McKeever P (2000) Relationships between nurses and family caregivers: Partners in Care? Advances in Nursing Science; 22(3):89-103

WHO (World Health Organization) (2008) Regional Office for Europe. The solid facts. Home Care in Europe. Edited by Tarricone R \& Tsouros AD. WHO Europe, Kopenhagen

Zeman P (1997) Häusliche Pflegearrangements. Interaktionsprobleme und Kooperationsperspektiven von lebensweltlichen und professionellen Hilfesystemen. In: Braun U, Schmidt R (Hrsg) Entwicklung einer lebensweltlichen Pflegekultur. Transfer-Verlag, Regensburg, S 97-112

ZQP (2013) (Zentrum für Qualität in der Pflege). Perspektivenwerkstatt 2013. ZQP-Fokus: Qualität in der häuslichen Pflege. ZQP, Berlin 